Note

\title{
Reciprocal Effects of Dietary Sesamin on Ketogenesis and Triacylglycerol Secretion by the Rat Liver
}

\author{
Nobuhiro Fukuda,,${ }^{1, *}$ Chika Miyagi, ${ }^{1}$ Lei Zhang, ${ }^{1}$ \\ Anura P. JAYasooriya, ${ }^{1}$ Masanobu SAKono, ${ }^{1}$ \\ Kyosuke YAmamoto, ${ }^{2}$ Takashi $\operatorname{IDE}^{3}$ \\ and Michihiro SugAnO ${ }^{4}$ \\ ${ }^{1}$ Department of Biological Resource Sciences, Faculty of Agriculture, \\ Miyazaki University, Miyazaki 889-2192, Japan \\ ${ }^{2}$ Department of Internal Medicine, Saga Medical University \\ School of Medicine, Saga 849-8501, Japan \\ ${ }^{3}$ Laboratory of Nutrition Biochemistry, \\ National Food Research Institute, Ministry of Agriculture, \\ Fisheries and Forestry, Tsukuba 305-8642, Japan \\ ${ }^{4}$ Department of Human Life Sciences, Prefectural University of Kumamoto, \\ Kumamoto 862-8502, Japan
}

(Received June 5, 1998)

Summary The effects of dietary sesamin (a mixture of sesamin and episesamin, $1: 1, \mathrm{w} / \mathrm{w}$ ) on ketone body production and lipid secretion were studied in isolated perfused liver from rats given sesamin. Feeding sesamin at the dietary level of $0.2 \%$ from 14 to $16 \mathrm{~d}$ resulted in an enlargement of liver weight. Ketone body production was significantly elevated in the livers perfused with oleic acid in comparison with those perfused without an exogenous-free fatty acid, and sesamin feeding caused a stimulation of ketone body production, especially when exogenous oleic acid was provided. On the other hand, the ratio of $\beta$-hydroxybutyrate to acetoacetate, an index of mitochondrial redox potential, tended to increase in the livers perfused with oleic acid compared with those without fatty acid, though it was consistently lowered by dietary sesamin. The cumulative secretion of triacylglycerol, but not of cholesterol, by the livers from sesamin-fed rats was decreased markedly, especially when exogenous oleic acid was provided, suggesting an inverse relationship between the rates of ketogenesis and triacylglycerol secretion. These results suggest that dietary sesamin exerts its hypotriglyceridemic effect at least in part through an enhanced metabolism of exogenous-free fatty acid to oxidation at the expense of esterification in rat liver.

Key Words sesamin, triacylglycerol secretion, ketone body production, perfused rat liver, hypotriglyceridemic effect

* To whom correspondence should be addressed. 
Dietary sesamin, one of the lignans existing most abundantly in sesame seeds and oils, has been shown to influence the metabolism of cholesterol and triacylglycerol, and also of polyunsaturated fatty acids (1-8). Furthermore, sesamin transiently enlarges liver weight accompanying a concomitant elevation of phospholipid levels $(1,2,4,7,8)$, which is similar to the effects observed in some hypolipidemic drugs such as fibrate and its related drugs, which are a potent hepatic peroxisomal proliferator (9-14). It has been suggested that the hypotriglyceridemic effect of these drugs is due to an increased partition of fatty acids to the pathways of oxidation at the expense of esterification by proliferating hepatic peroxisomes $(9,11-14)$. Ashakumary et al recently reported that sesamin causes a marked proliferation of hepatic peroxisomes and thus enhances the fatty acid oxidation rate in these organelles (15).

These observations therefore suggest that sesamin, like fibrate and its related drugs, may also influence alternative pathways of oxidation and esterification in rat liver. However, little is known about the effects of this lignan on the interactions between these two pathways. It is therefore of interest to examine whether dietary sesamin influences the partition of fatty acids between the pathways of oxidation and esterification in the liver. In the present study, we report for the first time that dietary sesamin enhances ketone body production and decreases triacylglycerol secretion in rat liver.

\section{Materials and methods}

Male Wistar rats (Kyudo, Kumamoto) weighing 140-150g were housed individually at a constant temperature $\left(22-24^{\circ} \mathrm{C}\right)$ with lights turned on from 07:00 to 19:00. They were acclimated to this situation for several days, then divided into two groups with equal body weights; one group was fed a control diet consisting of $94.5 \%$ powdered commercial chow (Type CE-2, Nihon Clea, Tokyo, Japan) and $5.5 \%$ corn oil, and the other group was fed the same diet supplemented with $0.2 \%$ sesamin (sesamin : episesamin, 1: 1, w/w, supplied by Suntory, Tokyo, Japan). These diets were given ad libitum for 14 to $16 \mathrm{~d}$. Food intake and body weight were recorded every other day.

On the day of the perfusion experiments, the rats were given intraperitoneal injections of pentobarbital $\mathrm{Na}(5 \mathrm{mg} / 100 \mathrm{~g}$ body weight $)$ at about 08:30. The liver was then isolated and perfused with $120 \mathrm{~mL}$ of recirculating Krebs-Henseleit perfusion medium ( $\mathrm{pH} 7.4$ ), which contained $25 \mathrm{~mm}$ glucose, $1.5 \%(\mathrm{w} / \mathrm{v})$ bovine serum albumin (Fraction V, Boehringer Mannheim), and 25\% (v/v) washed bovine erythrocytes, at a rate of $20 \mathrm{~mL} / \mathrm{min}$ at $37^{\circ} \mathrm{C}$ in the presence or absence of exogenous oleic acid. When livers were perfused with exogenous fatty acid, $5 \mathrm{~mL}$ of $20 \mathrm{~mm}$ potassium oleate $(100 \mu \mathrm{mol})$ were added at the beginning of recirculation, and the same solution was continuously infused at the rate of $4.5 \mathrm{~mL} / \mathrm{h}(90 \mu \mathrm{mol} / \mathrm{h})$. Every hour, $20 \mathrm{~mL}$ of perfusate were removed for analyses of ketone bodies and lipids, and the same amount of fresh perfusion medium was added to maintain $120 \mathrm{~mL}$ of recirculating volume. The perfusions were continued for $4 \mathrm{~h}$, as previously 
Table 1. Body weight, liver weight, and postperfused liver lipid.

\begin{tabular}{lccccc}
\hline & $\begin{array}{c}\text { Body } \\
\text { weight } \\
(\mathrm{g})\end{array}$ & $\begin{array}{c}\text { Liver weight* } \\
(\mathrm{g} / 100 \mathrm{~g} \text { body weight })\end{array}$ & \multicolumn{2}{c}{ Liver lipid $(\mu \mathrm{mol} / \mathrm{g})$} \\
\cline { 4 - 6 } & & & & & \\
& & & & & \\
\hline
\end{tabular}

Mean \pm SE of 4 rats. * Liver weight was measured at the end of perfusion. Values bearing different letters are significantly different at $p<0.05$. Rats weighing $140-150 \mathrm{~g}$ were fed the diets supplemented with or without $0.2 \%$ sesamin for 14 to $16 \mathrm{~d}$. The livers were then isolated and perfused in the presence or absence of an oleic acid substrate.

described in detail $(14,16,17)$. All aspects of the experiment were approved by the Miyazaki University Animal Policy and Welfare Committee.

The methods employed for the lipid and ketone body analyses in perfusates and postperfused livers were described previousiy $(14,16-18)$. Data were analyzed by ANOVA, followed by Duncan's new multiple-range test to evaluate significant differences of the means (19).

\section{Results and discussion}

Dietary sesamin did not influence food intake (23-25 g per d) or the growth of rats, although it did enlarge liver weight significantly (Table 1). No effects of dietary sesamin on lipid concentrations of the postperfused liver were observed, except for a significant increase in phospholipid levels of the livers perfused in the presence of exogenous oleic acid. The rate of synthesis and of secretion of triacylglycerol and cholesterol is influenced by the quality and quantity of exogenous fatty acids added in the perfusion medium $(16,20,21)$. Goh et al and Kohout et al found that oleic acid is the best substrate for the synthesis and secretion of triacylglycerol and cholesterol by the isolated rat liver perfused with equimolar quantities of palmitic, oleic, or linoleic acid, although the rate of hepatic uptake of free fatty acid was similar for all fatty acid substrates $(20,21)$. In the present study, we therefore employed oleic acid as an exogenous fatty acid substrate and found that the calculated values of hepatic uptake of oleic acid $(14,16,17)$ at consecutive time intervals are comparable: $183 \pm 3,272 \pm 4,361 \pm 3$, and $453 \pm 3 \mu \mathrm{mol} /$ liver in the control group, and $185 \pm 4,273 \pm 3,363 \pm 5$, and $454 \pm 5 \mu \mathrm{mol} /$ liver in the sesamin group, respectively. Therefore the following observations on the inverse responses in ketone body production and triacylglycerol 
Table 2. Effect of sesamin feeding on the cumulative production of ketone bodies and the ratio of $\beta$-hydroxybutyrate and acetoacetate.

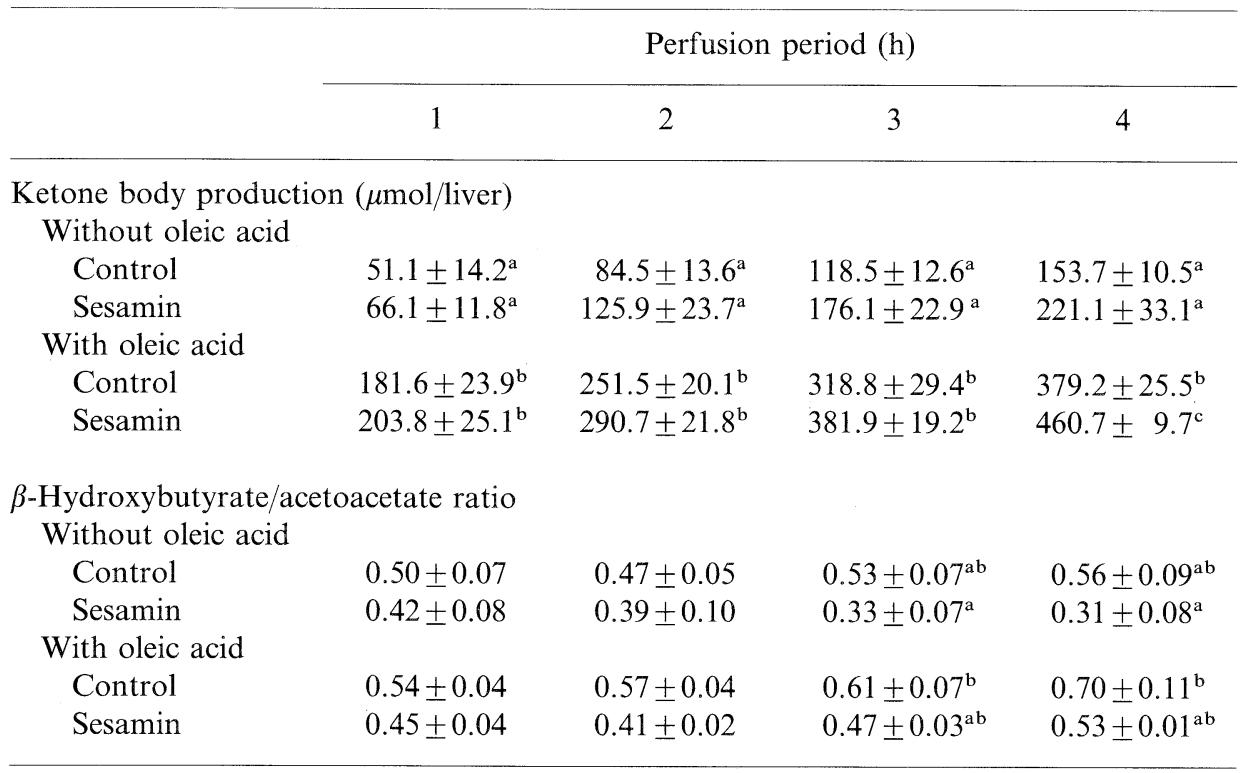

Mean \pm SE of 4 rats. Values bearing different letters are significantly different at $p<0.05$.

secretion by the livers in the presence of an exogenous fatty acid substrate may be attributed to the influences of dietary sesamin per se.

It has been reported that the elevation of ketone body production by livers perfused with an exogenous oleic acid substrate compared with those without the fatty acid substrate is usually accompanied by an increased ratio of $\beta$-hydroxybutyrate to acetoacetate because of an increased transportation of fatty acids across the mitochondrial membrane for $\beta$-oxidation $(14,17)$. Consistent with these observations, the present experiment showed that an infusion of an exogenous oleic acid substrate to the perfusion medium causes a 2.5 -fold increase in the production of ketone bodies with a concomitant elevation of the ratio of $\beta$-hydroxybutyrate to acetoacetate (Table 2). On the other hand, the livers of rats fed the sesamin diet produced more ketone bodies than animals fed the diet without the lignan did, both in the presence and in the absence of an oleic acid substrate, although statistical significance was observed only when an oleic acid was provided. In contrast, the ratio of $\beta$-hydroxybutyrate to acetoacetate was consistently lowered in the liver perfusates of the sesamin groups compared with those of the respective control groups, regardless of the presence or absence of exogenous-free fatty acid. These results suggest that the exogenous oleic acid substrate and dietary sesamin differently influence the redox potential in the liver mitochondrial compartment. Although the exact mechanism underlying the reciprocal responses in ketogenesis and the ratio of $\beta$-hydroxybutyrate to acetoacetate caused by dietary sesamin 
Table 3. Effect of sesamin feeding on the cumulative secretion of triacylglycerol and cholesterol by the perfused liver.

\begin{tabular}{|c|c|c|c|c|}
\hline & \multicolumn{4}{|c|}{ Perfusion period (h) } \\
\hline & 1 & 2 & 3 & 4 \\
\hline \multicolumn{5}{|c|}{ Triacylglycerol secretion ( $\mu \mathrm{mol} /$ liver $)$} \\
\hline \multicolumn{5}{|c|}{ Without oleic acid } \\
\hline Control & $3.2 \pm 0.3^{\mathrm{a}}$ & $6.8 \pm 0.4^{\mathrm{a}}$ & $9.9 \pm 0.6^{\mathrm{ac}}$ & $12.4 \pm 1.2^{\mathrm{a}}$ \\
\hline Sesamin & $1.5 \pm 0.5^{\mathrm{a}}$ & $3.2 \pm 0.9^{\mathrm{b}}$ & $7.5 \pm 1.9^{\mathrm{a}}$ & $12.8 \pm 3.9^{\mathrm{a}}$ \\
\hline \multicolumn{5}{|c|}{ With oleic acid } \\
\hline Control & $6.7 \pm 0.7^{\mathrm{a}}$ & $15.4 \pm 0.9^{c}$ & $24.7 \pm 2.4^{\mathrm{b}}$ & $36.4 \pm 5.5^{b}$ \\
\hline Sesamin & $3.4 \pm 0.6^{\mathrm{a}}$ & $8.9 \pm 0.9^{\mathrm{a}}$ & $15.1 \pm 1.4^{\mathrm{c}}$ & $22.0 \pm 2.6^{\mathrm{a}}$ \\
\hline \multicolumn{5}{|c|}{ Cholesterol secretion ( $\mu \mathrm{mol} /$ liver $)$} \\
\hline \multicolumn{5}{|c|}{ Without oleic acid } \\
\hline Control & $2.8 \pm 0.4$ & $3.5 \pm 0.3$ & $4.9 \pm 0.4$ & $6.1 \pm 0.6$ \\
\hline Sesamin & $2.2 \pm 0.3$ & $3.3 \pm 0.3$ & $5.5 \pm 0.4$ & $6.6 \pm 0.7$ \\
\hline \multicolumn{5}{|c|}{ With oleic acid } \\
\hline Control & $3.6 \pm 0.6$ & $5.6 \pm 0.6$ & $7.6 \pm 0.8$ & $10.0 \pm 0.4$ \\
\hline Sesamin & $2.9 \pm 0.5$ & $5.5 \pm 0.8$ & $7.6 \pm 0.9$ & $9.8 \pm 0.8$ \\
\hline
\end{tabular}

Mean \pm SE of 4 rats. Values bearing different letters are significantly different at $p<0.05$.

remains unclear, it has been reported that the feeding of clofibrate and its related drugs, a potent peroxisomal proliferator and a hypolipidemic drug, causes an enhanced ketone body production and decreases the ratio of $\beta$-hydroxybutyrate to acetoacetate $(13,14)$. Furthermore, these types of drugs cause an enlargement of liver weight accompanying a concomitant increase in the concentration of hepatic phospholipid and an inhibition of elongation and desaturation of polyunsaturated fatty acids (11-14). These observations suggest that the changes in various parameters caused by sesamin are related to the proliferation of hepatic peroxisomes. Ashakumary et al found that dietary sesamin causes a marked enhancement of hepatic fatty acid oxidation in peroxisomes and to a lesser extent in mitochondria, suggesting that sesamin, though structurally unrelated to clofibrate and its related drugs, also induces the proliferation of hepatic peroxisomes (15). These results indicate the existence of a common mechanism relating to the proliferation of peroxisomes, which may be playing an important role in an altered metabolism of fatty acids.

The infusion of an exogenous oleic acid substrate stimulated the hepatic secretion of triacylglycerol and cholesterol by 1.6 -fold and 1.5 -fold, respectively (Table 3). The increases in these two lipid molecules were both statistically significant, suggesting that free fatty acid supplied exogenously is a stimulus for the synthesis and secretion of triacylglycerol and cholesterol, consistent with previous 
observations $(14,17)$. On the other hand, sesamin feeding significantly attenuated the fatty acid dependent increase in hepatic triacylglycerol secretion by $40 \%$, but it remained unchanged in the absence of exogenous fatty acid. The lack of a reduction in triacylglycerol secretion in the latter perfusion condition appeared to be due to a reduced availability of endogenous fatty acid substrates used by the liver for the formation and secretion of triacylglycerol. These results suggest that the hypotriglyceridemic effect of dietary sesamin is in part attributed to a decreased hepatic synthesis and secretion of triacylglycerol. The mechanism(s) responsible for the observed reduction in the hepatic secretion of triacylglycerol by dietary sesamin is unclear. However, it has been reported that the reduced secretion of triacylglycerol by the livers of rats treated with fibrate and its related drugs is closely related to the drug-induced proliferation of hepatic peroxisomes $(11-14)$. Thus the serum triacylglycerol lowering effect of dietary sesamin can in part be associated with the proliferation of hepatic peroxisomes, primarily by diverting the exogenous fatty acid substrate to the peroxisomal fatty acid oxidation process. This leads to the reduced participation of exogenous substrate for the synthesis and secretion of triacylglycerol. It is of interest to compare the effects of sesamin and fibric acid derivatives on fatty acid metabolism, since these two chemicals are structurally unrelated to each other.

No difference was observed in the control and sesamin-fed rat livers in the cumulative secretion of cholesterol, regardless of the presence or absence of exogenous oleic acid. It has been reported that the hypocholesterolemic effect of sesamin is due to an inhibition of HMG-CoA reductase activity, the rate-limiting enzyme for cholesterol synthesis, in the liver in vivo (2). The decreased intestinal absorption of cholesterol by dietary sesamin has also been implicated for the serum cholesterol lowering effect in the rat (2). Thus the effect of this lignan is partly attributed to extrahepatic contributions such as an inhibitory effect of intestinal cholesterol absorption, an increased removal rate of cholesterol through LDL receptors, or a combination of these mechanisms besides the partial inhibition of cholesterol synthesis in the liver.

It has been shown that episesamin is produced from naturally occurring sesamin during the refining process of edible sesame oils (22). Shimizu et al found that sesamin and episesamin are potent and specific inhibitors of mammalian and microbial $\Delta 5$ desaturase involved in polyunsaturated fatty acid biosynthesis in vitro, but the magnitude of the inhibition by sesamin was far greater than that of the latter epimer (6). These observations suggest that the altered hepatic metabolism of free fatty acid caused by the feeding of a mixture of sesamin and episesamin in the present study is attributed to the effect of sesamin and not to the effect of episesamin. More detailed studies are required to distinguish the physiological activity of these two types of lignans.

In conclusion, a decreased secretion of triacylglycerol-rich lipoproteins by the liver from sesamin-fed rats is a consequence of a selective increase in ketone body production from exogenous fatty acids. 


\section{REFERENCES}

1) Sugano M, Inoue T, Koba K, Yoshida K, Hirose N, Shinmen Y, Akimoto K. 1990. Influence of sesame lignans on various lipid parameters in rats. Agric Biol Chem 54: 2669-2673.

2) Hirose Y, Inoue T, Nishihara K, Sugano M, Akimoto K, Shimizu S, Yamada H. 1991. Inhibition of cholesterol absorption and synthesis in rats by sesamin. $J$ Lipid Res 32: 629-638.

3) Akimoto K, Kitagawa Y, Akamatsu T, Hirose N, Sugano M, Shimizu S, Yamada H. 1993. Protective effects of sesamin against liver damage caused by alcohol or carbon tetrachloride in rodents. Ann Nutr Metab 37: 218-224.

4) Gu J-Y, Wakizono Y, Tsujita A, Lim B-O, Nonaka M, Yamada K, Sugano M. 1995. Effects of sesamin and $\alpha$-tocopherol, individually or in combination, on the polyunsaturated fatty acid metabolism, chemical mediator production, and immunoglobulin levels in Sprague-Dawley rats. Biosci Biotech Biochem 59: 2198-2202.

5) Hirata F, Fujita K, Ishikura Y, Hosoda K, Ishikawa T, Nakamura H. 1996. Hypocholesterolemic effect of sesame lignan in humans. Atherosclerosis 122: 135-136.

6) Shimizu S, Akimoto K, Shinmen Y, Kawashima H, Sugano M, Yamada H. 1991. Sesamin is a potent and specific inhibitor of 45 desaturase in polyunsaturated fatty acid biosynthesis. Lipids 26: 512-516.

7) Fujiyama-Fujiwara Y, Umeda-Sawada R, Kuzuyama M, Igarashi O. 1995. Effects of sesamin on the fatty acid composition of the liver of rats fed $n-6$ and $n-3$ fatty acid-rich diet. J Nutr Sci Vitaminol 41: 217-225.

8) Umeda-Sawada R, Takahashi N, Igarashi O. 1995. Interaction of sesamin and eicosapentaenoic acid against $\Delta 5$ desaturation and $n-6 / n-3$ ratio of essential fatty acids in rat. Biosci Biotech Biochem 59: 2268-2273.

9) Lazarow PB. 1977. Three hypolipidemic drugs increase hepatic palmitoyl-CoA oxidation in the rat. Science 197: 580-581.

10) Henninger C, Clouet P, Danh HC, Pascal M, Bezard J. 1987. Effects of fenofibrate treatment to fatty acid oxidation in liver mitochondria of obese Zucker rats. Biochem Pharmacol 36: 3231-3236.

11) Sanchez RM, Vinals M, Alegret M, Vazquez M, Adzet T, Merlos M, Laguna JC. 1993. Fibrates modify rat hepatic fatty acid chain elongation and desaturation in vitro. Biochem Pharmacol 46: 1791-1796.

12) Cohen AJ. 1981. Review of the hepatic response to hypolipidemic drugs in rodent and assessment of its toxicological significance to man. Fd Cosmet Toxicol 19: 585-605.

13) Laker ME, Mayes PA. 1979. The immediate and long term effects of clofibrate on the metabolism of the perfused rat liver. Biochem Pharmacol 28: 2813-2827.

14) Yamamoto K, Fukuda N, Zhang L, Sakai T. 1996. Altered hepatic metabolism of fatty acids in rats fed a hypolipidemic drug, fenofibrate. Pharmacol Res 33: 337-342.

15) Ashakumary L, Rouyer I, Ide T, Fukuda N, Sugano M. 1998. Reciprocal responses to sesamin of the gene expression and activity of enzyme in fatty acid oxidation and synthesis in rat liver. The 52nd Annual Meeting of Nippon Eiyo Shokuryou Gakkai $(J$ Jpn Nutr Food Sci) (Okinawa), Abstract: 90.

16) Fukuda N, Azain MJ, Ontko JA. 1982. Altered hepatic metabolism of free fatty acids underlying hypersecretion of very low density lipoproteins in the genetically obese Zucker rat. J Biol Chem 257: 14066-14072.

17) Fukuda N, Ontko JA. 1984. Interactions between fatty acid synthesis, oxidation, and

Vol 44, No 5, 1998 
esterification in the production of triglyceride-rich lipoproteins by the liver. $J$ Lipid Res 25: 831-842.

18) Folch J, Lees M, Sloane-Stanley GH. 1957. A simple method for the isolation and purification of total lipids from animal tissues. $J$ Biol Chem 226: 497-509.

19) Duncan DB. 1955. Multiple range and multiple F tests. Biometrics 11: 1-42.

20) Kohout MK, Kouhoutova B, Heimberg M. 1971. The regulation of hepatic triglyceride metabolism by free fatty acids. $J$ Biol Chem 246: 5067-5074.

21) Goh E, Heimberg M. 1977. Effects of free fatty acids on activity of hepatic microsomal 3-hydroxy-3-methylglutaryl coenzyme A reductase and on secretion of triglyceride and cholesterol by liver. $J$ Biol Chem 252: 2822-2826.

22) Fukuda Y, Nagata M, Osawa T, Namiki M. 1986. Contribution of lignan analogues to antioxidant activity of refined unroasted sesame seed oil. JAOCS 63: 1027-1031. 